\title{
Therapeutic Plasma Exchange in Refractory Hyperthyroidism
}

\author{
Clotilde Saïe $^{\mathrm{a}}$ Cécile Ghander ${ }^{\mathrm{a}}$ Samir Saheb $^{\mathrm{b}}$ Christel Jublanc ${ }^{\mathrm{c}}$ \\ Denis Lemesle ${ }^{d}$ Charlotte Lussey-Lepoutre ${ }^{e}$ Laurence Leenhardt ${ }^{a}$ \\ Fabrice Menegaux ${ }^{f}$ Christophe Tresallet $^{g}$ Camille Buffet $^{a}$
}

a'Thyroid and Endocrine Tumors Unit, Pitié-Salpêtrière Hospital APHP, Sorbonne University, Paris, France;

${ }^{b}$ Apheresis Unit, Pitié-Salpêtrière Hospital, AP-HP, Sorbonne University, Paris, France; ${ }^{C}$ Department of

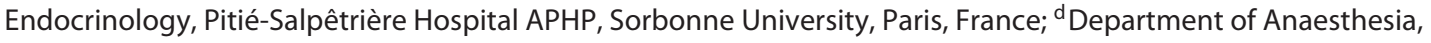

Pitié-Salpêtrière Hospital APHP, Sorbonne University, Paris, France; ${ }^{\mathrm{e} N u c l e a r}$ Medicine Department, Pitié-Salpêtrière

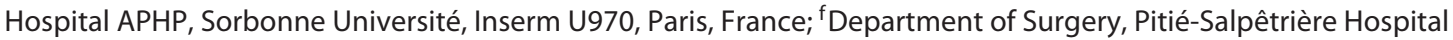
APHP, Sorbonne University, Paris, France; ' Department of Surgery, Avicennes Hospital, Paris, France

\section{Keywords}

Therapeutic plasma exchange $\cdot$ Thyrotoxicosis $\cdot$ Thyroid surgery $\cdot$ lodine-induced thyrotoxicosis $\cdot$ Hyperthyroidism

\begin{abstract}
Introduction: Hyperthyroid patients who are unresponsive to medical treatment remain a challenging clinical problem.

Objective: The goal of our study was to evaluate the use of therapeutic plasma exchange (TPE) in hyperthyroid patients and their outcome after TPE. Method: We retrospectively reviewed 22 patients who underwent TPE for refractory thyrotoxicosis in our institution: 13 with Graves' disease, 7 with amiodarone-induced thyrotoxicosis (AIT), 1 with toxic goiter, and 1 pregnant patient with familial nonautoimmune thyrotoxicosis. Results: Before TPE, all patients had severe hyperthyroidism, and antithyroid drugs were either contraindicated or not sufficiently effective to restore euthyroidism promptly. After all the TPEs, free $\mathrm{T}_{4}$ (fT4) decreased significantly by $48 \%(p=0.001)$ and fT3 by $52 \%(p=0.0001)$. The median number of TPE sessions per patient was 4 (range: 1-10). There were no complications during the 91 TPE sessions. Total thyroidectomy with no severe side effects was
\end{abstract}

karger@karger.com www.karger.com/etj

(C) 2020 European Thyroid Association Published by S. Karger AG, Basel performed on $16 / 22$ patients and 1 other patient was treated with radioactive iodine. One patient died from severe thyrotoxicosis during medical care. The remaining 4 patients were followed up without any radical treatment. For all 7 patients with AIT, iterative TPE led to a significant clinical improvement, and amiodarone was continued for 1 patient. Available treatments were continued between TPE sessions (cholestyramine for 13 patients [60\%] and glucocorticoids for 16 patients [73\%]). Conclusion: TPE allowed a safe decrease of $50 \%$ in thyroid hormone levels, and it should be considered for refractory hyperthyroid patients when medical treatments are contraindicated or have failed to restore euthyroidism, irrespective of the etiology of the thyrotoxicosis.

(C) 2020 European Thyroid Association Published by S. Karger AG, Basel

\section{Introduction}

Severe thyrotoxicosis is a life-threatening condition, especially when patients have heart disease or atrial fibrillation [1]. The main objective is to promptly restore and maintain euthyroidism. Therapeutic plasma exchange (TPE) is an effective blood purification procedure used to 
treat various disorders such as autoimmune diseases and familial hypercholesterolemia [2].

In hyperthyroidism, TPE has been used since 1970 [3] and is mainly considered for use in clinical practice for the following conditions:

- in thyroid storm, which is a vital emergency requiring a rapid decrease in thyroid hormone levels [4],

- in severe thyrotoxicosis for patients with heart disease or severe cardiac rhythm disorder, as an alternative to medical treatment which may have a delayed action [5],

- for patients with severe hyperthyroidism who have a contraindication to antithyroid drugs (ATD), due to severe side effects such as agranulocytosis, to restore euthyroidism before any radical treatment of hyperthyroidism (i.e., thyroidectomy or radioiodine treatment) [1].

We report a retrospective series of 22 hyperthyroid patients who underwent TPE in our tertiary care center hospital. Our goals were to describe the use of TPE in refractory hyperthyroidism, the conditions under which the sessions were carried out, and to analyze the potential complications of this treatment. We also focused on the outcome of patients after the TPE sessions. In the case of salvage thyroidectomy performed after TPE preparation, peri- and postoperative complications related to anesthesia or surgery were also analyzed.

\section{Materials and Methods}

All patients treated with TPE in our tertiary care center between 2007 and the first semester of 2017 were retrospectively included. Twenty-two patients were included (Table 1). There were 10 men (44\%) and 12 women (56\%) with a median age of 47 years (range 16-85 years). Graves' disease (GD) was diagnosed in 13 patients (59\%), 7 patients (32\%) had amiodarone-induced thyrotoxicosis (AIT), and 1 patient had a toxic goiter. The remaining case was a pregnant woman in whom the cause of thyrotoxicosis was an activating mutation of the thyroid-stimulating hormone (TSH) receptor (nonautoimmune thyrotoxicosis). In the patients with GD, the median TSH receptor antibody level was $16 \mathrm{U} / \mathrm{L}$ (range 9-41 U/L). All patients with AIT had type 2 AIT, confirmed by ${ }^{123}$-iodine thyroid scintigraphy (online suppl. Fig.; www.karger. com/doi/10.1159/000507019) or histology (patient No. 13). They also all had a history of heart disease: 4 had arrhythmia, 2 had congenital heart defects, and 1 had ischemic heart disease.

An indication for TPE was a contraindication to ATD due to major side effects in 13 patients, namely, a profound decrease in neutrophils in 7 ( 5 due to carbimazole and 2 to thiamazole), propylthiouracil (PTU)-induced hepatotoxicity in 5 (including 1 who also presented with agranulocytosis due to carbimazole and 1 who had cutaneous toxidermia to carbimazole), thrombopenia in 1 , and a severe cutaneous reaction in 1 . All of these side effects disappeared after discontinuing ATD. Another indication was the failure to control hyperthyroidism in 9 patients on medical treatment, defined as patients who presented with high thyroid hormone levels despite an adequate length of treatment and adequate doses of ATD. This was decided at a multidisciplinary staff meeting at which the clinical situation and comorbidities were considered. Adequate treatment was based on guideline recommendations, i.e., $400 \mathrm{mg}$ PTU and $30 \mathrm{mg}$ methylprednisone over 4 or 6 weeks for AIT [5], and $50 \mathrm{mg}$ glucocorticoids for GD [6].

TPE was performed in either the Apheresis Unit or the intensive care unit. TPE consisted of removing one and a half volumes of plasma during each session and replacing this with a solution of $5 \%$ albumin in saline. Consecutive plasma exchanges were conducted using the Spectra Optia (BCT Terumo). The decision to start the treatment and the number of sessions required were agreed on at a meeting attended by the endocrinologist, surgeon, and apheresis specialists.

The following criteria were analyzed: the etiology of thyrotoxicosis; clinical features; the levels of free tri-iodothyronine (fT3) (ref. range 2.20-4.70 pmol/L), free thyroxine (fT4) (ref. range 11$26 \mathrm{pmol} / \mathrm{L}$ ), thyroid-stimulating hormone (TSH) (ref. range 0.4$4 \mathrm{mUI} / \mathrm{L}$ ), and TSH receptor antibody; the number of sessions and their side effects; and the drugs administered in parallel with TPE. Following TPE, we collected all analyses of fT3, fT4, and TSH performed the same day until 11 days after TPE. TSH receptor antibodies after TPE were not available. Finally, we studied the outcome of patients at the end of the TPE sessions, including thyroidectomy and radioiodine treatment data.

The fT3, fT4, and TSH measurements were analyzed at the Endocrine Biochemistry Department of our hospital using an electrochemiluminescence $\left(\right.$ Roche $\left.^{\circledR}\right)$ assay.

\section{Statistical Analysis}

Analyses were performed using Prism 7, with $p<0.05$ regarded as statistically significant. For group comparisons, the Student $t$ test was used to analyze quantitative variables. The $\chi^{2}$ test was used for qualitative variables.

\section{Results}

Before TPE, all patients presented with severe thyrotoxicosis, according to their medical records. Median fT4 concentration was $68 \mathrm{pmol} / \mathrm{L}$ (range 30.7-100] and median $\mathrm{fT} 3$ concentration was $21 \mathrm{pmol} / \mathrm{L}$ (range 6.6-48.2).

Available treatments were continued between TPE sessions. Cholestyramine was given to 13 patients $(60 \%)$ at a mean dose of $10 \mathrm{~g}$ (range $4-12 \mathrm{~g}$ ). Twelve patients (54\%) were given PTU at a mean dose of $430 \mathrm{mg}$ (range 300-600 $\mathrm{mg}$ ), for a mean period of 4 weeks (range 1-8 weeks). Regarding glucocorticoids, 16 patients (73\%) were treated with a mean dose of $55 \mathrm{mg}$ prednisone (range $40-80 \mathrm{mg}$ ); a similar mean dose of $50 \mathrm{mg}$ (range: $50-60 \mathrm{mg}$ ) was given to AIT patients and GD patients received $53.6 \mathrm{mg}$ (range 40 $80 \mathrm{mg}$ ). All were treated for an average of 5 weeks before TPE (range 1-15 weeks). In accordance with current guidelines, most of them were treated with $\beta$-blockers (70\%). 


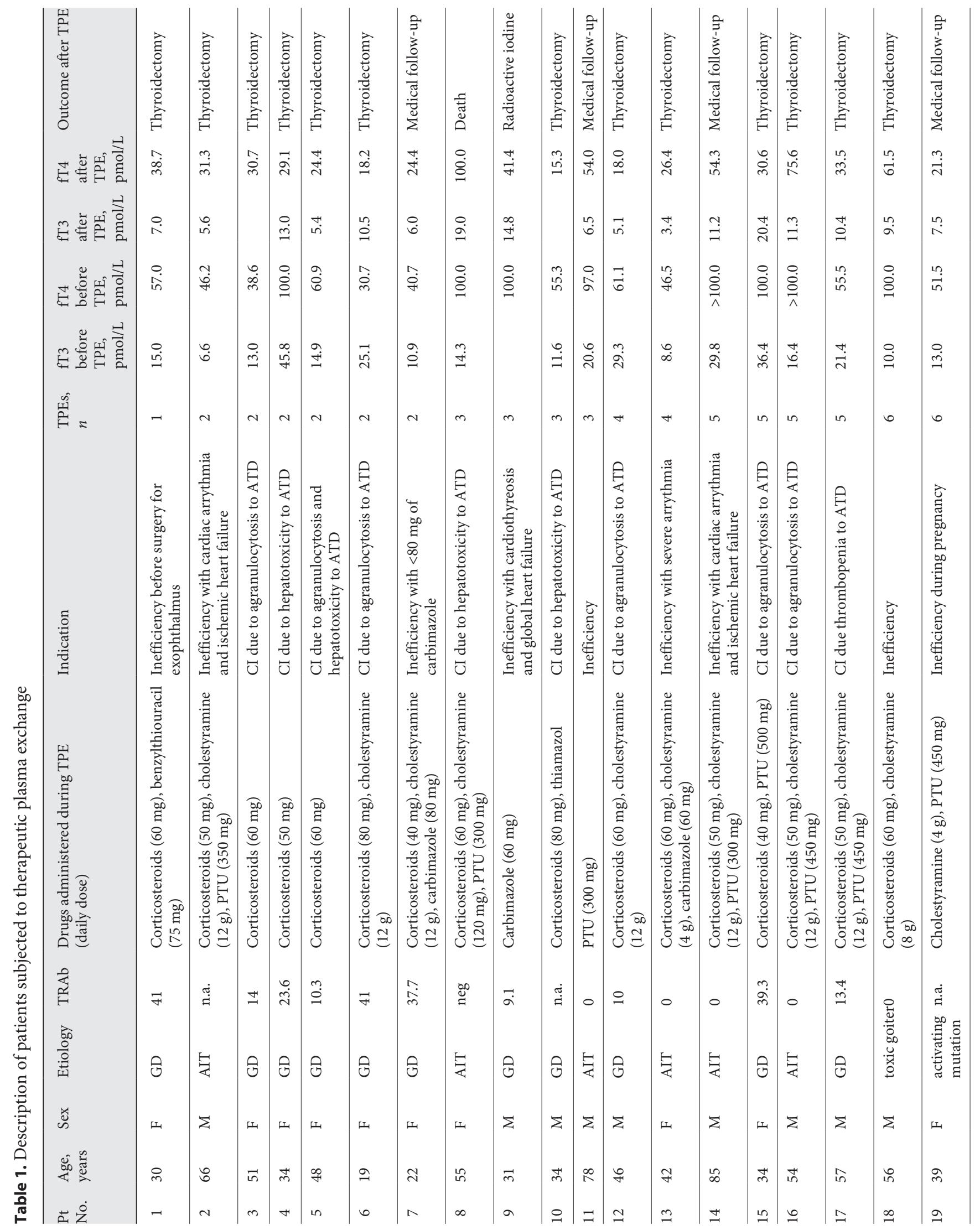




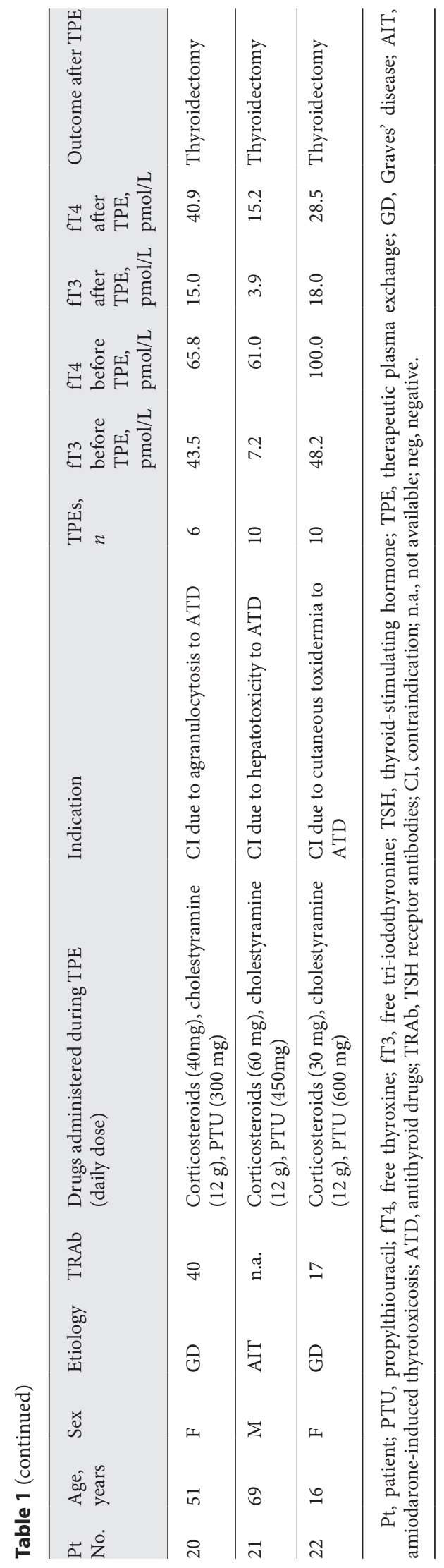

Plasma Exchange in Hyperthyroidism

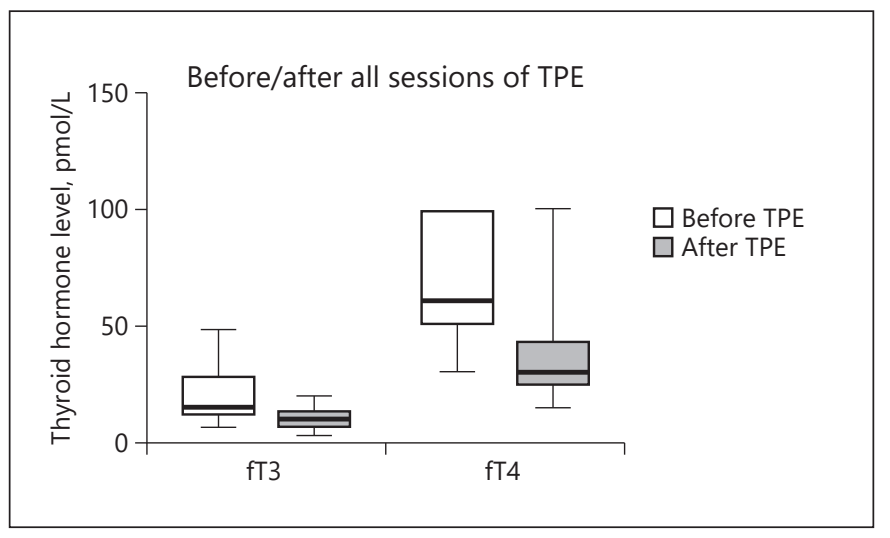

Fig. 1. Levels of fT3 and fT4 before and after all sessions of TPE. Box, interquartile range with median; whiskers, range. fT4, free thyroxine; fT3, free tri-iodothyronine; TPE, therapeutic plasma exchange.

\section{Description and Impact of TPE}

In total, we reviewed 91 TPEs in 22 patients. The mean time between diagnosis and the first TPE session was 3 months (range $<1-26$ months). For GD and AIT patients, mean time between diagnosis and the first TPE were, respectively, 4 (range $<1-26)$ and $2(<1-13)$ months. The period between 2 sessions was between 1 and 8 days $(2.7$ days on average).

The first hormonal measurement was performed between 1 and $10 \mathrm{~h}$ after the TPE session, and it showed a $20 \%$ decrease in fT4 and fT 3 from the pre-TPE level. fT4 and fT 3 levels were measured, on average, 2 days (range $0-11$ days) after all TPE sessions. fT4 decreased significantly by $48 \%(p=0.001)$ and fT3 by $52 \%(p=0.001)$ (Fig. 1). TPE failed to decrease thyroid hormone levels in 1 case (patient No. 8) despite 3 sessions. TPE restored fT3 to normal levels in 2 patients (9\%) in an average time of 3 days. fT4 levels were strictly normalized in 8 patients (36\%) within an average of 6 days (range 1-19 days). The normalization of both fT 3 and fT 4 was achieved in 1 patient.

Patients had between 1 and 10 TPE sessions. The average number of TPEs per patient was 4 (range 1-10). More than 4 TPEs were needed for 9 patients. As described in Table 2, there were no factors that predicted when $>4$ TPEs would be needed. The 2 groups were similar regarding sex $(p=0.43)$, age $(p=0.3)$, and etiology. The median level of TSH receptor antibodies $(<3.3 \mathrm{U} / \mathrm{L})$ was $17 \mathrm{U} / \mathrm{L}$ in patients receiving $<4$ TPEs and $15 \mathrm{U} / \mathrm{L}$ in patients receiving $>4$ TPEs $(p=0.87)$. There was also no significant difference between AIT and GD patients regarding indications or thyroid hormone levels (Table 2). 
Table 2. Comparison of patients by number of TPE received and by etiology

\begin{tabular}{|c|c|c|c|c|c|c|}
\hline Patients, $n$ & 13 & 9 & & 13 & 7 & \\
\hline Females, $n$ & 8 & 4 & 0.43 & 9 & 2 & 0.08 \\
\hline Age, years & 42 & 51 & 0.3 & 37 & 64 & 0.06 \\
\hline $\mathrm{GD}, n$ & 9 & 4 & 0.24 & - & - & - \\
\hline AIT, $n$ & 4 & 3 & 0.88 & - & - & - \\
\hline Contraindication of ATD, $n$ & 7 & 6 & 0.54 & 10 & 3 & 0.12 \\
\hline Inefficiency of ATD, $n$ & 6 & 2 & 0.25 & 3 & 4 & 0.12 \\
\hline Mean time between diagnosis and the first TPE session, months & 6 & 4.6 & 0.3 & 4 & 2 & 0.16 \\
\hline fT3 before TPE, pmol/L & 17 & 25 & 0.22 & 26 & 15 & 0.06 \\
\hline fT4 before TPE, pmol/L & 64 & 81 & 0.11 & 67 & 79 & 0.1 \\
\hline fT3 after all TPE sessions, pmol/L & 8.7 & 11.9 & 0.17 & 12 & 8.7 & 0.14 \\
\hline
\end{tabular}

TPE, therapeutic plasma exchange; GD, Graves' disease; AIT, amiodarone-induced thyrotoxicosis; ATD, antithyroid drugs.

No complications, e.g., hypotension and catheterrelated complications (hematoma, infection, or thrombosis) were noted either during or after the TPE sessions. At the end of the iterative TPE sessions, no hypocalcemia was observed. The median decrease in hemoglobin was $1 \mathrm{~g} / \mathrm{dL} ; 4$ patients showed a significant decrease $(>3 \mathrm{~g} / \mathrm{dL})$ but none of them required a transfusion.

\section{Outcome after TPE Procedure}

Definitive treatments were performed for 17 patients (77\%) after at least 1 TPE session: 16 thyroidectomies (11 GD and 4 AIT patients and the patient with a toxic multinodular goiter), and 1 radioiodine treatment (1 GD patient).

Regarding surgical procedures, heavy perioperative bleeding was reported by the surgeon for 3 patients with GD, but no transfusion was necessary. There were no anesthetic complications. All patients were hemodynamically stable without the use of any vasoactive drugs. The heart rate was controlled thanks to $\beta$-blockers. Postoperatively, hypocalcemia was detected in 3 patients (total calcium 1.53-1.93 $\mathrm{mmol} / \mathrm{L}$ [ref. range $2.2-2.6 \mathrm{mmol} / \mathrm{L}$ ]). Transitory dysphonia was observed in 1 patient. No histopathological malignancy was detected in the 16 cases that underwent surgery.

One patient was treated with radioactive iodine (355 MegaBequerel), which led to hypothyroidism without any other side effects. Severe hyperthyroidism due to AIT combined with heart failure led to the death of a 55-yearold woman (Table 1, patient No. 8) with dilated cardiomyopathy who was awaiting heart transplantation, despite therapeutic management including corticosteroids, cholestyramine, PTU, and 3 TPE sessions.

In 4 patients ( $1 \mathrm{GD}$ and 2 AIT patients and 1 with an activating mutation of the TSH receptor), TPE sessions associated with medication improved their clinical state and a simple clinical follow-up was possible without any radical treatment.

\section{Discussion}

For the clinician, the management of hyperthyroid patients is challenging when ATD fail. AIT can be a dangerous condition as it can exacerbate underlying cardiac diseases and increase morbidity and mortality. The current European Thyroid Association (ETA) guidelines for the management of amiodarone-associated thyroid dysfunction recommend emergency treatment, particularly for the elderly and/or in cases of reduced left ventricular function [5]. TPE is reported to be useful, despite the lack of evidence-based medicine. In our study on 22 patients, including 7 with AIT, TPE allowed a rapid decrease, approximately $50 \%$, in fT 3 and fT4 levels; this led to improvement in all but 1 patient. This result is consistent
90

Eur Thyroid J 2021;10:86-92 DOI: $10.1159 / 000507019$
Saïe et al. 
with a recent publication reporting TPE in 46 thyrotoxic patients including 4 with AIT [7].

TPE was found to be a good preparation for surgery in our study, as there were no surgical or anesthetic complications recorded even in patients with a history of heart disease. In a previous study, the reported surgical morbidity rate was $29 \%$ in patients operated on for AIT without any TPE preparation [8]. In our study, hypocalcemia was recorded in 3 patients (19\%) after thyroidectomy, which corresponds to the reported incidence of transitory postoperative hypocalcemia in the literature $[9,10]$. Ozbey et al. [11] raised the awareness of the decrease in antithrombotic factors and thus the risk of perioperative bleeding in thyroid surgery after TPE. In 3 thyroidectomies after TPE for GD, surgeons recorded perioperative bleeding, but there were no transfusions or adverse anesthetic events.

Furthermore, therapeutic management including TPE sufficiently decreased fT 3 and fT 4 levels to avoid any radical treatment in 2 AIT patients, and enabled amiodarone to be continued in 1 . In the 6 other patients with AIT, amiodarone was stopped by the cardiologist. The ETA guidelines for the management of amiodarone-associated thyroid dysfunction state that there is no sufficient evidence to recommend for or against amiodarone withdrawal [5]. Medical management including TPE permitted the rapid control of severe hyperthyroidism or definitive treatment to be undertaken without major complications in $21 / 22$ patients. Our results are similar to another study on 22 patients in which only 1 patient died (as in our series) [12].

TPE is a safe treatment. Indeed, during the 91 sessions, no side effects were observed, even in patients with a history of stroke $(n=2)$ or heart failure $(n=4)$ or 1 with a transplanted liver. Compared to other series $[7,11]$, no hypocalcemia was induced by TPE in our cohort. In the study by Simsir et al. [7], hemoglobin levels were significantly decreased. In 4 of our patients, hemoglobin levels dropped but no blood transfusion was necessary. There is no absolute contraindication described in the literature, and in our experience none of the clinical situations contraindicated the procedure. For example, patient No. 6 had a history of ischemic stroke 3 months before conducting the TPE sessions. Despite the risk of low flow and the aggravation of neurological symptoms, TPE treatment was approved by the neurologists and the sessions were carried out without any complications or neurological aggravation. The TPEs were also safe in the pregnant patient in our cohort and in 2 pregnant patients in Simsir et al. [7].

Plasma Exchange in Hyperthyroidism
From a pathophysiological point of view, each TPE session consists of removing plasma, which contains components such as protein-bound hormones. The replacement with albumin solution brings new binding sites for free thyroid hormones leading, to a decrease in fT3 and fT4 levels [13]. In the case of GD, a reduction in TSH receptor-stimulating antibodies can also play a role. Unfortunately, information regarding the evolution of antibody levels before and after TPE was not available. A decrease in TSH receptor antibodies after 4 TPEs has been described in a case report of GD with an ATD contraindication [14], but information is lacking in other published cohort studies $[7,11,12,15]$.

Due to the severity of thyrotoxicosis in our series, medical treatments against thyrotoxicosis were continued between TPE sessions, if not contraindicated to increase the chances of promptly restoring euthyroidism. Glucocorticoids, ATD, and cholestyramine were often prescribed in parallel with TPE, as in other studies [11, 12].

One limit of our study is the lack of comparison between patients subjected to TPE and patients with medical treatment alone without TPE. To formally demonstrate that TPE is beneficial, a randomized study comparing 2 strategies with or without TPE for comparable clinical situations, in terms of the etiology and severity of hyperthyroidism, would provide valuable information. However, given the heterogeneity of the patients (i.e., an etiology of hyperthyroidism and comorbidities) in such severe clinical conditions, randomized studies would be difficult to implement. Nevertheless, this study underlines the efficacy of TPE associated with medical treatments available to control severe thyrotoxicosis.

The median number of TPE sessions was 4 , as in other series $[7,11,12]$. By comparing patients who received more or fewer than 4 TPEs sessions, we found no predictive factors to determine the number of TPEs required. Accordingly, we believe that this should be decided on a case-by-case basis and reassessed after each session by clinicians.

TPE is not currently recommended for the treatment of hyperthyroidism [1]. Indeed radioiodine, surgery, and ATD are sufficient in most cases. However, TPEs can be useful in AIT cases with heart failure. TPEs are also proposed in the case of thyroid storm [2] since their rapid action can be welcome for this life-threating situation. Muller et al. [4] reported successful TPEs for 3 patients with thyroid storm. Moreover, when patients have had a serious allergic reaction to ATD, the recom- 
mendation is to discontinue this therapeutic class. In this situation, we believe that TPEs may contribute to the preparation for a radical treatment in the case of severe hyperthyroidism.

Our study is limited by the heterogeneity of the data analyzed due to its retrospective nature. The results regarding TPE efficacy are, on the whole, significant, although individual variability cannot be denied. However, our results outline that TPE associated with medical treatment permits a safe decrease $(50 \%)$ in thyroid hormone levels, even in patients with severe comorbidities. Its efficiency is linked to the etiology of hyperthyroidism and could be used for AIT patients, in particular. TPE could be an alternative treatment in some difficult clinical situations, for instance, to prepare for radical treatment.

\section{Acknowledgements}

We thank Natacha Jumentier and Fatima Karcha for their careful management of the patients reported in this series and Liz Atzel for editing the English version.

\section{Statement of Ethics}

This retrospective study was ethically approved by the Thyroid and Endocrine Tumors Research Group ("Groupe de Recherche Clinique-Tumeurs Thyroïdiennes" at the Sorbonne University). Oral consent was obtained from each patient after full explanation of the purpose and nature of all procedures used, particularly TPE.

\section{Disclosure Statement}

The authors have no conflicts of interest to declare.

\section{Funding Sources}

This research did not receive any specific grant from any funding agency in the public, commercial, or not-for-profit sector.

\section{Author Contributions}

C.S.: conception of the work and acquisition and analysis of data. C.G.: conception of the work. S.S., C.J., D.L., C.L.-L., F.M., and C.T.: critical revision for important intellectual content and final approval of the version to be published. L.L.: conception of the work, revising it critically for important intellectual content, and final approval of the version to be published. C.B.: conception of the work, analysis of data, critical revision for important intellectual content, and final approval of the version to be published.

\section{References}

1 Bahn Chair RS, Burch HB, Cooper DS, Garber JR, Greenlee MC, Klein I, et al.; American Association of Clinical Endocrinologists. Hyperthyroidism and other causes of thyrotoxicosis: management guidelines of the American Thyroid Association and American Association of Clinical Endocrinologists. Thyroid. 2011 Jun;21(6):593-646.

2 Schwartz J, Padmanabhan A, Aqui N, Balogun RA, Connelly-Smith L, Delaney M, et al. Guidelines on the Use of Therapeutic Apheresis in Clinical Practice-Evidence-Based Approach from the Writing Committee of the American Society for Apheresis: The Seventh Special Issue. J Clin Apher. 2016 Jun;31(3): $149-62$.

3 Ashkar FS, Katims RB, Smoak WM 3rd, Gilson AJ. Thyroid storm treatment with blood exchange and plasmapheresis. JAMA. 1970 Nov;214(7):1275-9.

4 Muller C, Perrin P, Faller B, Richter S, Chantrel F. Role of plasma exchange in the thyroid storm. Ther Apher Dial. 2011 Dec;15(6):52231.

5 Bartalena L, Bogazzi F, Chiovato L, Hubalewska-Dydejczyk A, Links TP, Vanderpump M. 2018 European Thyroid Associa- tion (ETA) Guidelines for the Management of Amiodarone-Associated Thyroid Dysfunction. Eur Thyroid J. 2018 Mar;7(2):55-66.

6 Kahaly GJ, Bartalena L, Hegedüs L, Leenhardt L, Poppe K, Pearce SH. 2018 European Thyroid Association Guideline for the Management of Graves' Hyperthyroidism. Eur Thyroid J. 2018 Aug;7(4):167-86.

7 Simsir IY, Ozdemir M, Duman S, Erdogan M, Donmez A, Ozgen AG. Therapeutic plasmapheresis in thyrotoxic patients. Endocrine. 2018 Oct;62(1):144-8.

8 Houghton SG, Farley DR, Brennan MD, van Heerden JA, Thompson GB, Grant CS. Surgical management of amiodarone-associated thyrotoxicosis: mayo Clinic experience. World J Surg. 2004 Nov;28(11):1083-7.

9 Hussain M, Hisham AN. Total thyroidectomy: the procedure of choice for toxic goitre. Asian J Surg. 2008 Apr;31(2):59-62.

10 Sywak MS, Palazzo FF, Yeh M, Wilkinson M, Snook K, Sidhu SB, et al. Parathyroid hormone assay predicts hypocalcaemia after total thyroidectomy. ANZ J Surg. 2007 Aug;77(8): 667-70.

11 Ozbey N, Kalayoglu-Besisik S, Gul N, Bozbora A, Sencer E, Molvalilar S. Therapeutic plas- mapheresis in patients with severe hyperthyroidism in whom antithyroid drugs are contraindicated. Int J Clin Pract. 2004 Jun;58(6): 554-8.

12 Keklik M, Kaynar L, Yilmaz M, Sivgin S, Solmaz M, Pala C, et al. The results of therapeutic plasma exchange in patients with severe hyperthyroidism: a retrospective multicenter study. Transfus Apheresis Sci. 2013 Jun;48(3):327-30.

13 Schlienger JL, Faradji A, Demangeat C, Sapin R, Chabrier G, Simon C, et al. Quantitative evaluation of hormonal extraction performed by continuous plasma exchange in euthyroid patients. Application to the treatment of severe hyperthyroidism. Presse Med. 1983; 12(8):499-502.

14 Miljić D, Stojanović M, Ješić R, Bogadnović G, Popović V. Role of plasma exchange in autoimmune hyperthyroidism complicated by severe tiamazol-induced cholestatic jaundice. Transfus Apheresis Sci. 2013 Oct;49(2):3546.

15 Ezer A, Caliskan K, Parlakgumus A, Belli S, Kozanoglu I, Yildirim S. Preoperative therapeutic plasma exchange in patients with thyrotoxicosis. J Clin Apher. 2009;24(3):111-4. 